\title{
Radiative Lifetimes for Singly Ionized Beryllium
}

\author{
G. $\operatorname{ÇeliK}^{a, *}$, B. Atalay ${ }^{b}$ And Ş. AteŞ ${ }^{a}$ \\ ${ }^{a}$ Department of Physics, Faculty of Science, Selçuk University, Campus 42049 Konya, Turkey \\ ${ }^{b}$ Department of Physics, Faculty of Arts and Sciences, Canakkale Onsekiz Mart University, Canakkale, Turkey
}

(Received February 9, 2016; in final form June 28, 2016)

\begin{abstract}
The theoretical lifetimes for singly ionized beryllium (Be II) have been calculated using the weakest bound electron potential model theory and the quantum defect orbital theory under the assumption of the $L S$ coupling scheme. In the calculations, many states are considered. Some lifetime values for highly excited levels have been obtained using these methods. The lifetimes presented in this work have been compared with the early theoretical calculations and measurements presented in the literature. A good agreement with the results in the literature has been obtained.
\end{abstract}

DOI: 10.12693/APhysPolA.130.705

PACS/topics: 31.15.ag, 32.70.Cs

\section{Introduction}

The lifetime is one of the fundamental characteristics of excited states of atoms and ions. Measurements of lifetime of atomic and ionic states are of constant interest in different branches of physics such as astrophysics, space physics, plasma physics and atomic physics [1,2]. When the electromagnetic interaction in atomic physics is well understood, the radiative lifetimes give some valuable information about atomic structure [3]. This information extracted from atomic lifetime calculations and measurements can be of relevance to a wide variety of areas from astrophysics to lamp design. Singly ionized beryllium, an ion of an alkaline-earth element, is also of importance in many study fields such as astrophysics, theoretical and experimental atomic physics. Because of not being formed in conventional stellar nucleosynthesis but rather being generated by high-energy reactions between heavy nuclei and cosmic rays or protons from interstellar matter, beryllium ions are especially important in astrophysics [4]. Therefore, lifetime measurements and calculations in singly-ionized beryllium are attractive for theoretical and experimental researches.

In the present paper, we report lifetime calculations in Li-like beryllium. The calculations are carried out using the weakest bound electron potential model (WBEPM) theory and the quantum defect orbital (QDO) theory. In the calculations the $n s(n=3-10), n p(n=2-10), n d$ $(n=3-9)$ and $n f(n=4-10)$ states for both multiplets and individual lines have been considered in Be II.

\section{Theory and calculation procedure}

The electric dipole transition probability (in $\mathrm{s}^{-1}$ ) from a level labeled by $n l L J$ quantum numbers to another level labeled by $n^{\prime} l^{\prime} L^{\prime} J^{\prime}$ quantum numbers is given to be [5]:

*corresponding author; e-mail: gultekin@selcuk.edu.tr

$$
\begin{gathered}
A_{n l L J, n^{\prime} l^{\prime} L^{\prime} J^{\prime}}=\frac{64 \pi^{4} e^{2} a_{0}^{2}\left(E_{J^{\prime}}-E_{J}\right)^{3}}{3 h(2 J+1)} S_{E 1}= \\
2.0261 \times 10^{-6} \frac{\left(E_{J^{\prime}}-E_{J}\right)^{3}}{(2 J+1)} S_{E 1},
\end{gathered}
$$

where $e$ is the electron charge, $a_{0}$ is the Bohr radius, $h$ is the Planck constant, $\left(E_{J^{\prime}}-E_{J}\right)$ is the transition energy in Kaysers $\left(\mathrm{cm}^{-1}\right)$ units and $(2 J+1)$ is the degeneracy of initial level and $S_{E 1}$ is the electric dipole line strength in atomic units.

The lifetime of a given level is calculated to

$$
\tau_{J^{\prime}}=\frac{1}{\sum_{J} A_{J^{\prime} J}},
$$

where $A_{J^{\prime} J}$ is the transition probability and the summation holds for all levels $J\left(E_{J}<E_{J^{\prime}}\right)$ radiatively connected to the level $J^{\prime}$.

Fundamental quantity for the calculation of transition probabilities, oscillator strengths and lifetimes of excited levels is the $S_{J J^{\prime}}$ line strength. The line strength is determined according to the coupling schemes and the transition types in atomic or ionic systems. The line strength expression includes transition integrals, angular coefficients and transition energies. In this study, we have employed both the WBEPM theory and the QDO theory in order to calculate radial transition integrals in the determination of lifetimes for Be II.

\subsection{The WBEPM theory}

According to the WBEPM theory, electrons in a system can be divided into two groups of electrons to be the weakest bound electron (WBE) and the non-weakest bound electrons (NWBE). The WBE in a given manyelectron system is an electron excited or ionized by the least energy to the system compared to the other electrons in the system. In this method, the electronic radial wave function can be expressed by means of generalized Laguerre polynomial depending on some parameters. These parameters are determined from experimental energy data and the expectation values of radii for levels. The many of atomic or ionic processes such as ionization, 
excitation and rates of radiative decay may be related to the WBE. The properties of WBE give accurate information about these processes. In this method, according to WBE approximation, many-electron problem can be simplified as a single electron problem [6-10].

In the WBEPM theory framework, the radial wave function of the WBE [9] can be obtained to be

$$
\begin{aligned}
& R_{n^{*} l^{*}}(r)=\left(\frac{2 Z^{*}}{n^{*}}\right)^{l^{*}+3 / 2}\left[\frac{2 n^{*}}{(n-l-1) !} \Gamma\left(n^{*}+l^{*}+1\right)\right]^{-1 / 2} \\
& \quad \times \exp \left(-\frac{Z^{*} r}{n^{*}}\right) r^{l^{*}} L_{n-l-1}^{2 l^{*}+1}\left(\frac{2 Z^{*} r}{n^{*}}\right) .
\end{aligned}
$$

In Eq. (3), $L_{n-l-1}^{2 l^{*}+1}\left(\frac{2 Z^{*} r}{n^{*}}\right)$ are the generalized Laguerre polynomials, $\Gamma(x)$ is a gamma function [9]. $Z *, n *, l^{*}$ parameters can be obtained by solving Eq. (4) and Eq. (5) together

$$
\begin{aligned}
& I=-\varepsilon=\frac{Z *^{2}}{2 n *^{2}}, \\
& \langle r\rangle=\frac{3 n *^{2}-l *(l *+1)}{2 Z *},
\end{aligned}
$$

where $I$ is ionization energy and $\langle r\rangle$ is expectation value for radius of weakest bound electron. The ionization energies and expectation values for radii of all states must be known for $Z *, n *, l^{*}$ parameters to be determined.

\subsection{The QDO theory}

The QDO theory is based on analytical solution of the one-electron Schrödinger equation with an effective central-field potential [11]. This formalism gives exact solutions for the radial orbital and analytical expressions for transition integrals. Moreover, it possesses other advantages as it contains a screening parameter in its model potential that is formulated in terms of experimental data, therefore, correlation and even relativistic effects are implicitly accounted for.

The QDO theory involves the exact solution of the radial equation with the potential

$$
V_{r}=\frac{\lambda(\lambda+1)-l(l+1)}{2 r^{2}}-\frac{z_{\text {net }}}{r} .
$$

In this equation, $Z_{\text {net }}$ is the effective nuclear charge at large radial distances, $l$ is the angular-momentum quantum number, and $\lambda$, which also appears in the quantum defect radial orbitals, is generally determined as

$$
\lambda=l-\delta+c,
$$

where $\delta$ is the quantum defect ( $\delta$ depends on effective principal quantum numbers with $n^{*}=n-\delta$ ) obtained from empirical atomic level data [12] given as

$$
E=-Z_{\text {net }}^{2} / 2 n^{* 2} \text {. }
$$

Moreover, $c$ is an integer and the range of permitted values of $c$ is limited by the conditions on the parameter $\lambda$ as follows:

(1) The radial wave function must be quadratically integrable, for which

$$
\lambda \leq n-\delta-1, \quad \lambda \geq-1
$$

(2) expectation values of the energy must be potentially evaluable, for which

$$
\lambda>-1 / 2 \text {. }
$$

These two conditions lead to the following range of variation of $c[13]$ :

$$
\delta-l-(1 / 2)<c \leq n-l-1 .
$$

A large number of chemically important properties of atoms and molecules can be theoretically evaluated, if the appropriate valence, Rydberg, or continuum orbitals are known. The Schrödinger equation for the radial part of a valence, Rydberg, or continuum orbital in the centralfield approximation is given as [13]:

$$
-\frac{1}{2} \frac{\mathrm{d}^{2} \mu}{\mathrm{d} r^{2}}+\left[\frac{l(l+1)}{2 r^{2}}+V(r)\right] \mu=E \mu .
$$

The appropriate bound-state wave functions are given to be

$$
\mu(r)=W_{n-\delta, \lambda+1 / 2}\left[2 Z_{\text {net }} r /(n-\delta)\right],
$$

where $W$ is the second Whittaker function. The normalized bound-state orbitals may be expressed in terms of generalized Laguerre polynomials or in terms of Kummer's functions [13]:

$$
\begin{gathered}
\mu(r)=\left[\frac{Z_{\text {net }} \Gamma\left(n^{*}+\lambda+1\right)}{\Gamma\left(n^{*}+\lambda\right)}\right]^{1 / 2}\left[\frac{\left(2 Z_{\text {net }} r / n^{*}\right)^{\lambda+1}}{n^{*} \Gamma(2 \lambda+2)}\right] \\
\times \mathrm{e}^{-Z_{\text {net }} r / n^{*}} F\left[\lambda+1-n+\delta, 2 \lambda+2,2 Z_{\text {net }} r / n^{*}\right] .
\end{gathered}
$$

The radial transition integrals $R_{i j}$ are

$$
R_{i j}=\int \mu_{i}(r) r \mu_{j}(r) r^{2} \mathrm{~d} r
$$

by substituting the bound-state orbital functions of Eq. (14) into Eq. (15).

\section{Results and discussion}

In this paper, the lifetimes of excited levels have been calculated using the WBEPM theory and the QDO theory for many of states of lithium like beryllium. In the WBEPM theory, the $Z^{*}, n^{*}$ and $l^{*}$ parameters are required for the calculations. These parameters are determined using the energy expressions and the expectation values of radii of states. The required energy values have been taken from NIST [14]. The wave functions in the QDO theory are obtained by solving the one-electron Schrödinger equation with the approximate central-field electron-screening potential. In the determination of wave functions, $n^{*}, \lambda$ parameters and $\delta$ quantum defect parameter are required. In the previous papers, we presented how to obtain these parameters in detail [15-20].

We have calculated the lifetime values of highly excited levels for Be II and the obtained results are given in Table I. The last lines of each set of states given as Mlt in Table I represent the lifetimes of multiplet lines. Our lifetime results have been compared with some theoretical results obtained from the relativistic many-body calculations using a high-precision relativistic all-order method which includes all single, double, and partial 
triple excitations of the Dirac-Fock wave functions given by Safranova et al. [21], the multiconfiguration HartreeFock + Breit-Pauli $(\mathrm{MCHF}+\mathrm{BP})$ method results given by Froese Fischer et al. [22], the compilation of calculated lifetimes using a numerical Coulomb approximation with a Hartree-Slater core (CAHS) approach given by Theodosiou [23], the Fues model potential (FMP) approach calculations given by Glukhov et al. [24, 25], the presented values in the Grotrian database [26], the numerical Coulomb approximation results given by Lindgard et al. [27], with some experimental measurement values given by Bromander [28], Hontzeas et al. [29] and Andersen et al. [30]. The experimental results are given together with their uncertainty rating. Moreover, our lifetime results have been compared with the semiempirical calculations given by Kurucz [31].

Lifetimes (ns) of excited levels for singly ionized beryllium.

TABLE I

\begin{tabular}{|c|c|c|c|c|c|c|c|c|c|c|c|c|c|}
\hline \multirow{3}{*}{ Levels } & \multirow{3}{*}{ Term } & \multirow{3}{*}{$J$} & \multirow{3}{*}{$\begin{array}{l}\text { Energy } \\
{\left[\mathrm{cm}^{-1}\right]}\end{array}$} & \multicolumn{2}{|c|}{ This work } & \multirow{2}{*}{\multicolumn{7}{|c|}{ Ref. }} & \multirow{3}{*}{$\begin{array}{l}\text { Exp. } \\
\text { results }\end{array}$} \\
\hline & & & & \multirow{2}{*}{$\begin{array}{c}\text { WBEPM } \\
\text { theory }\end{array}$} & \multirow{2}{*}{$\begin{array}{c}\text { QDO } \\
\text { theory }\end{array}$} & & & & & & & & \\
\hline & & & & & & [21] & [24] & [23] & {$[26]$} & {$[22]$} & {$[27]$} & [31] & \\
\hline $1 s(2) 2 p(1)$ & ${ }^{2} P$ & 1.5 & 31935.320 & 8.64 & 8.51 & $8.85(2)$ & & & & 8.8471 & & 8.621 & \\
\hline $1 s(2) 2 p(1)$ & ${ }^{2} P$ & 0.5 & 31928.744 & 8.64 & 8.50 & $8.85(2)$ & & & & 8.8528 & & 8.621 & \\
\hline $1 s(2) 2 p(1)$ & ${ }^{2} P$ & Mlt & 31933.127 & 8.64 & 8.50 & & 8.504 & 8.93 & 8.9 & & 8.939 & & $\begin{array}{l}9.5(2)^{c}, \\
8.1(4)^{e}\end{array}$ \\
\hline $1 s(2) 3 p(1)$ & ${ }^{2} P$ & 1.5 & 96497.288 & 5.56 & 5.52 & $5.417(2)$ & & & & 5.4159 & & 4.149 & \\
\hline $1 s(2) 3 p(1)$ & ${ }^{2} P$ & 0.5 & 96495.360 & 5.18 & 5.52 & $5.417(1)$ & & & & 5.4181 & & 4.149 & \\
\hline $1 s(2) 3 p(1)$ & ${ }^{2} P$ & Mlt & 96496.645 & 5.56 & 5.52 & & 5.522 & 5.431 & 5.6 & & 5.615 & & $6.7(8)^{d}$ \\
\hline $1 s(2) 4 p(1)$ & ${ }^{2} P$ & 1.5 & 118761.320 & 8.07 & 8.23 & $8.086(1)$ & & & & & & 6.211 & \\
\hline $1 s(2) 4 p(1)$ & ${ }^{2} P$ & 0.5 & 118760.510 & 8.07 & 8.23 & $8.072(3)$ & & & & & & 6.211 & \\
\hline $1 s(2) 4 p(1)$ & ${ }^{2} P$ & Mlt & 118760.050 & 8.07 & 8.23 & & 8.232 & 8.03 & 8.3 & & 8.312 & & $8.5(9)^{d}$ \\
\hline $1 s(2) 5 p(1)$ & ${ }^{2} P$ & 1.5 & 128972.050 & 13.1 & 13.2 & $12.918(4)$ & & & & & & 9.901 & \\
\hline $1 s(2) 5 p(1)$ & ${ }^{2} P$ & 0.5 & 128971.620 & 13.1 & 13.2 & $12.935(6)$ & & & & & & 9.901 & \\
\hline $1 s(2) 5 p(1)$ & ${ }^{2} P$ & Mlt & 128971.910 & 13.1 & 13.2 & & 13.15 & 12.76 & 13.5 & & & & \\
\hline $1 s(2) 6 p(1)$ & ${ }^{2} P$ & 1.5 & 134485.610 & 20.6 & 20.4 & $20.05(1)$ & & & & & & 15.432 & \\
\hline $1 s(2) 6 p(1)$ & ${ }^{2} P$ & 0.5 & 134485.370 & 20.6 & 20.4 & $20.04(2)$ & & & & & & 15.432 & \\
\hline $1 s(2) 6 p(1)$ & ${ }^{2} P$ & Mlt & 134485.530 & 20.6 & 20.4 & & 20.39 & 19.71 & 20.5 & & & & \\
\hline $1 s(2) 7 p(1)$ & ${ }^{2} P$ & 1.5 & 137796.120 & 30.8 & 30.3 & $29.78(2)$ & & & & & & 22.936 & \\
\hline $1 s(2) 7 p(1)$ & ${ }^{2} P$ & 0.5 & 137795.970 & 30.8 & 30.3 & $29.78(3)$ & & & & & & 22.936 & \\
\hline $1 s(2) 7 p(1)$ & ${ }^{2} P$ & $M l t$ & 137796.070 & 30.8 & 30.3 & & 30.29 & 29.19 & 30.5 & & & & \\
\hline $1 s(2) 8 p(1)$ & ${ }^{2} P$ & 1.5 & 139938.180 & 44.1 & 43.3 & $42.66(4)$ & & & & & & 32.680 & \\
\hline $1 s(2) 8 p(1)$ & ${ }^{2} P$ & 0.5 & 139938.080 & 44.1 & 43.3 & $42.47(5)$ & & & & & & 32.680 & \\
\hline $1 s(2) 8 p(1)$ & ${ }^{2} P$ & $M l t$ & 139938.150 & 44.2 & 43.3 & & 43.25 & 41.57 & 43.5 & & & & \\
\hline $1 s(2) 9 p(1)$ & ${ }^{2} P$ & 1.5 & 141403.310 & 61.1 & 59.7 & $58.86(7)$ & & & & & & 45.045 & \\
\hline $1 s(2) 9 p(1)$ & ${ }^{2} P$ & 0.5 & 141403.240 & 61.1 & 59.7 & $58.86(14)$ & & & & & & 45.045 & \\
\hline $1 s(2) 9 p(1)$ & ${ }^{2} P$ & $M l t$ & 141403.290 & 61.1 & 59.7 & & 59.69 & 57.25 & 60.1 & & & & \\
\hline $1 s(2) 10 p(1)$ & ${ }^{2} P$ & 1.5 & 142449.330 & 82.2 & 80.1 & $78.6(2)$ & & & & & & 60.606 & \\
\hline $1 s(2) 10 p(1)$ & ${ }^{2} P$ & 0.5 & 142449.300 & 82.2 & 80.1 & $78.9(7)$ & & & & & & 60.606 & \\
\hline $1 s(2) 10 p(1)$ & ${ }^{2} P$ & $M l t$ & 142449.330 & 82.2 & 80.1 & & 80.03 & 76.66 & 80.5 & & & & \\
\hline $1 s(2) 3 s(1)$ & ${ }^{2} S$ & Mlt & 88231.915 & 2.46 & 2.46 & $2.451(1)$ & 2.461 & 2.473 & 2.5 & 2.4497 & 2.511 & 2.545 & $2.3(3)^{c}$ \\
\hline $1 s(2) 4 s(1)$ & ${ }^{2} S$ & Mlt & 115464.440 & 4.24 & 4.20 & $4.174(1)$ & 4.199 & 4.196 & 4.3 & 4.1315 & 4.254 & 4.486 & $5.0(3)^{c}$ \\
\hline $1 s(2) 5 s(1)$ & ${ }^{2} S$ & Mlt & 127335.120 & 7.35 & 7.24 & $7.194(2)$ & 7.236 & 7.213 & 7.3 & & & 7.519 & \\
\hline $1 s(2) 6 s(1)$ & ${ }^{2} S$ & Mlt & 133556.440 & 12.0 & 11.8 & $11.673(2)$ & 11.76 & 11.70 & 12 & & & 12.210 & \\
\hline $1 s(2) 7 s(1)$ & ${ }^{2} S$ & Mlt & 137218.780 & 18.4 & 18.0 & $17.918(4)$ & 18.02 & 18.02 & 18.5 & & & 18.622 & \\
\hline $1 s(2) 8 s(1)$ & ${ }^{2} S$ & Mlt & 139555.160 & 25.9 & 26.4 & $26.17(1)$ & 26.34 & 26.22 & 26.5 & & & 27.174 & \\
\hline $1 s(2) 9 s(1)$ & ${ }^{2} S$ & Mlt & 141136.300 & 37.9 & 37.0 & $36.78(2)$ & 36.99 & 36.81 & 37.5 & & & 38.023 & \\
\hline $1 s(2) 10 s(1)$ & ${ }^{2} S$ & Mlt & 142255.840 & 51.5 & 50.3 & $49.89(7)$ & 50.27 & 50.02 & 51 & & & 51.546 & \\
\hline $1 s(2) 3 d(1)$ & ${ }^{2} D$ & 2.5 & 98055.100 & 0.892 & 0.862 & $0.901(1)$ & & & & 0.9045 & & 0.893 & \\
\hline $1 s(2) 3 d(1)$ & ${ }^{2} D$ & 1.5 & 98054.570 & 0.897 & 0.862 & $0.904(1)$ & & & & 0.9043 & & 0.893 & \\
\hline $1 s(2) 3 d(1)$ & ${ }^{2} D$ & Mlt & 98054.890 & 0.897 & 0.862 & & 0.862 & 0.911 & 0.9 & & 0.911 & & $0.92(6)^{c}$ \\
\hline
\end{tabular}


TABLE I cont.

Lifetimes (ns) of excited levels for singly ionized beryllium.

\begin{tabular}{|c|c|c|c|c|c|c|c|c|c|c|c|c|c|}
\hline \multirow{3}{*}{ Levels } & \multirow{3}{*}{ Term } & \multirow{3}{*}{$J$} & \multirow{3}{*}{$\begin{array}{l}\text { Energy } \\
{\left[\mathrm{cm}^{-1}\right]}\end{array}$} & \multicolumn{2}{|c|}{ This work } & \multirow{2}{*}{\multicolumn{7}{|c|}{ Ref. }} & \multirow{3}{*}{$\begin{array}{l}\text { Exp. } \\
\text { results }\end{array}$} \\
\hline & & & & \multirow{2}{*}{$\begin{array}{c}\text { WBEPM } \\
\text { theory }\end{array}$} & \multirow{2}{*}{$\begin{array}{c}\text { QDO } \\
\text { theory }\end{array}$} & & & & & & & & \\
\hline & & & & & & [21] & {$\left[{ }^{a} /{ }^{b}\right]$} & [23] & {$[26]$} & [22] & [27] & [31] & \\
\hline $1 s(2) 4 d(1)$ & ${ }^{2} D$ & 2.5 & 119421.440 & 2.02 & 1.99 & $2.066(1)$ & & & & & & 2.075 & \\
\hline $1 s(2) 4 d(1)$ & ${ }^{2} D$ & 1.5 & 119421.200 & 2.02 & 1.99 & $2.067(1)$ & & & & & & 2.075 & \\
\hline $1 s(2) 4 d(1)$ & ${ }^{2} D$ & Mlt & 119421.340 & 2.02 & 1.99 & & $1.991^{a}$ & 2.081 & 2.1 & & 2.069 & & $\begin{array}{l}2.12(9)^{c} \\
2.30(9)^{c}\end{array}$ \\
\hline $1 s(2) 5 d(1)$ & ${ }^{2} D$ & 2.5 & 129310.250 & 3.83 & 3.82 & $3.938(2)$ & & & & & & 3.984 & \\
\hline $1 s(2) 5 d(1)$ & ${ }^{2} D$ & 1.5 & 129310.130 & 3.83 & 3.82 & $3.950(1)$ & & & & & & 3.984 & \\
\hline $1 s(2) 5 d(1)$ & ${ }^{2} D$ & $M l t$ & 129310.200 & 3.83 & 3.82 & & $3.817^{a}$ & 3.971 & 3.9 & & 3.940 & & $3.5(4)^{d}$ \\
\hline $1 s(2) 6 d(1)$ & ${ }^{2} D$ & 2.5 & 134681.150 & 6.50 & 6.51 & $6.700(2)$ & & & & & & 6.757 & \\
\hline $1 s(2) 6 d(1)$ & ${ }^{2} D$ & 1.5 & 134681.150 & 6.51 & 6.51 & $6.701(2)$ & & & & & & 6.757 & \\
\hline $1 s(2) 6 d(1)$ & ${ }^{2} D$ & $M l t$ & 134681.150 & 6.51 & 6.51 & & $6.507^{a}$ & 6.754 & 6.7 & & 6.688 & & $7.3(8)^{d}$ \\
\hline $1 s(2) 7 d(1)$ & ${ }^{2} D$ & 2.5 & 137919.170 & 10.2 & 10.2 & $10.518(4)$ & & & & & & 10.650 & \\
\hline $1 s(2) 7 d(1)$ & ${ }^{2} D$ & 1.5 & 137919.170 & 10.2 & 10.2 & $10.522(1)$ & & & & & & 10.650 & \\
\hline $1 s(2) 7 d(1)$ & ${ }^{2} D$ & Mlt & 137919.170 & 10.2 & 10.2 & & $10.23^{a}$ & 10.61 & 10.5 & & & & \\
\hline $1 s(2) 8 d(1)$ & ${ }^{2} D$ & 2.5 & 140020.580 & 15.1 & 15.2 & $15.58(1)$ & & & & & & 15.773 & \\
\hline $1 s(2) 8 d(1)$ & ${ }^{2} D$ & 1.5 & 140020.580 & 15.1 & 15.2 & $15.58(1)$ & & & & & & 15.773 & \\
\hline $1 s(2) 8 d(1)$ & ${ }^{2} D$ & $M l t$ & 140020.580 & 15.1 & 15.2 & & $15.16^{a}$ & 15.71 & 15.5 & & & & \\
\hline $1 s(2) 9 d(1)$ & ${ }^{2} D$ & 2.5 & 141461.150 & 21.3 & 21.5 & $22.01(1)$ & & & & & & 22.321 & \\
\hline $1 s(2) 9 d(1)$ & ${ }^{2} D$ & 1.5 & 141461.150 & 21.3 & 21.5 & $22.05(1)$ & & & & & & 22.321 & \\
\hline $1 s(2) 9 d(1)$ & ${ }^{2} D$ & $M l t$ & 141461.150 & 21.3 & 21.5 & & $21.46^{a}$ & 22.22 & 22 & & & & \\
\hline $1 s(2) 4 f(1)$ & ${ }^{2} F$ & 3.5 & 119446.720 & 4.51 & 4.51 & $4.525(1)$ & & & & & & 4.505 & \\
\hline $1 s(2) 4 f(1)$ & ${ }^{2} F$ & 2.5 & 119446.590 & 4.51 & 4.51 & $4.531(1)$ & & & & & & 4.505 & \\
\hline $1 s(2) 4 f(1)$ & ${ }^{2} F$ & Mlt & 119446.660 & 4.51 & 4.51 & & $4.510^{b}$ & 4.522 & 4.5 & & 4.523 & & $\begin{array}{l}4.9(2)^{c}, \\
7.0(4)^{e}\end{array}$ \\
\hline $1 s(2) 5 f(1)$ & ${ }^{2} F$ & 3.5 & 129323.920 & 8.71 & 8.72 & $8.734(1)$ & & & & & & 8.772 & \\
\hline $1 s(2) 5 f(1)$ & ${ }^{2} F$ & 2.5 & 129323.850 & 8.71 & 8.72 & $8.739(2)$ & & & & & & 8.772 & \\
\hline $1 s(2) 5 f(1)$ & ${ }^{2} F$ & $M l t$ & 129323.890 & 8.71 & 8.72 & & $8.722^{b}$ & 8.739 & 8.8 & & 8.737 & & $9.0(8)^{d}$ \\
\hline $1 s(2) 6 f(1)$ & ${ }^{2} F$ & 3.5 & 134689.230 & 14.9 & 14.9 & $14.948(6)$ & & & & & & 14.948 & \\
\hline $1 s(2) 6 f(1)$ & ${ }^{2} F$ & 2.5 & 134689.200 & 14.9 & 14.9 & $14.959(6)$ & & & & & & 14.948 & \\
\hline $1 s(2) 6 f(1)$ & ${ }^{2} F$ & $M l t$ & 134689.220 & 14.9 & 14.9 & & $14.91^{b}$ & 14.93 & 15 & & 14.93 & & \\
\hline $1 s(2) 7 f(1)$ & ${ }^{2} F$ & 3.5 & 137924.310 & 23.4 & 23.5 & $23.60(1)$ & & & & & & 23.474 & \\
\hline $1 s(2) 7 f(1)$ & ${ }^{2} F$ & 2.5 & 137924.310 & 23.4 & 23.5 & $23.60(1)$ & & & & & & 23.474 & \\
\hline $1 s(2) 7 f(1)$ & ${ }^{2} F$ & Mlt & 137924.310 & 23.4 & 23.5 & & $23.45^{b}$ & 23.49 & 23.6 & & & & \\
\hline $1 s(2) 8 f(1)$ & ${ }^{2} F$ & 3.5 & 140024.120 & 34.7 & 34.7 & $35.03(1)$ & & & & & & 34.843 & \\
\hline $1 s(2) 8 f(1)$ & ${ }^{2} F$ & 2.5 & 140024.120 & 34.7 & 34.7 & $35.01(1)$ & & & & & & 34.843 & \\
\hline $1 s(2) 8 f(1)$ & ${ }^{2} F$ & Mlt & 140024.120 & 34.7 & 34.7 & & $34.72^{b}$ & 34.77 & 35 & & & & \\
\hline $1 s(2) 9 f(1)$ & ${ }^{2} F$ & 3.5 & 141463.650 & 49.1 & 49.1 & $49.73(2)$ & & & & & 49.261 & 49.261 & \\
\hline $1 s(2) 9 f(1)$ & ${ }^{2} F$ & 2.5 & 141463.650 & 49.1 & 49.1 & $49.71(2)$ & & & & & 49.261 & 49.261 & \\
\hline $1 s(2) 9 f(1)$ & ${ }^{2} F$ & Mlt & 141463.650 & 49.1 & 49.1 & & $49.11^{b}$ & 49.18 & 49.3 & & & & \\
\hline $1 s(2) 10 f(1)$ & ${ }^{2} F$ & 3.5 & 142493.330 & 66.9 & 66.9 & & & & & & 67.114 & 67.114 & \\
\hline $1 s(2) 10 f(1)$ & ${ }^{2} F$ & 2.5 & 142493.330 & 66.9 & 66.9 & & & & & & 67.114 & 67.114 & \\
\hline $1 s(2) 10 f(1)$ & ${ }^{2} F$ & Mlt & 142493.330 & 66.9 & 66.9 & & & & & & & & \\
\hline
\end{tabular}

As a result of comparisons, it can be seen from Table I that the average agreements of our lifetime values obtained by using the WBEPM theory and the QDO theory are $\pm 2-3 \%$ to other theoretical results [21-27], are $\pm 9 \%$ to the semi-empirical calculations given by $\mathrm{Ku}-$ rucz [31]. Experimental results are scarcely available for comparisons, especially for highly excited states. Since the observation of highly excited states is difficult, the experimental results for some states considered in this work are not available. When one considers the comparison values in Table I, both our results and other theoretical results given in the literature are in divergence from experimental values. Unfortunately, such deviations are appreciable in almost all states. But, the average agreements of our lifetime values are $\pm 6-20 \%$ to the experimental results [28-30] given in Table I. 


\section{Conclusions}

We list the lifetimes of the 85 levels in Table I. It can be seen from Table I that there are very good agreements between the present results and the previously published theoretical results. Although some discrepancies are seen with experimental results, they are all within the experimental uncertainty.

The structure of ions of group II elements such as Be II is quite similar to that of the alkali atoms. Therefore, in calculating transition probabilities, oscillator strengths and lifetimes it is reasonable to use the WBEPM theory and the QDO theory, which has been successfully applied to numerical calculations of the atomic structure parameters of alkali atoms and other multi-electron systems in previous decades [15-20]. These calculation methods are especially useful when the large numbers of transition probabilities are required, since wave functions and matrix elements are computed quickly and automatically using only energy level, ionization potential data and the expectation values of radii belonging to levels as inputs. The configuration interaction effects and the relativistic effects are considered partially through the use of the energy and the expectation values used in determination of required parameters in both the WBEPM theory and the QDO theory. In this work, some lifetimes of the higher excited levels are obtained using the WBEPM theory and the QDO theory through a more simple procedure.

\section{Acknowledgments}

The author gratefully acknowledges the Selçuk University Scientific Research Projects (BAP) Coordinating Office for support.

\section{References}

[1] L. Liang, Y.C. Wang, J. Phys. B At. Mol. Opt. Phys. 36, 4387 (2003).

[2] W.L. Wiese, Progress in Atomic Spectroscopy, Eds. W. Hanle, H. Kleinpoppen, Part B, Plenum, New York 1978

[3] E. Gomez, F. Baumer, A.D. Lange, G.D. Sprouse, Phys. Rev. A 72, 012502 (2005).

[4] H. Reeves, J.P. Meyer, Astrophys. J. 226, 613 (1978).

[5] R.D. Cowan, The Theory of Atomic Structure and Spectra, University of California Press, California 1981.

[6] N.W. Zheng, T. Wang, Astrophys. J. Suppl. Ser. 143, 231 (2002).
[7] N.W. Zheng, T. Wang, R.Y. Yang, T. Zhou, D.X. Ma, Y.G. Wu, H.T. Xu, At. Data Nucl. Data Tables $\mathbf{7 9}$ 109 (2001).

[8] N.W. Zheng, Y.J. Sun, T. Wang, D.X. Ma, Y. Zhang, W. Su, Int. J. Quant. Chem. 76, 51(2000).

[9] N.W. Zheng, T. Wang, R. Yang, Y.G. Wu, J. Chem. Phys. 112, 7042 (2000).

[10] N.W. Zheng, T. Wang, Chem. Phys. Lett. 376, 557 (2003).

[11] G. Simons, J. Chem. Phys. 55, 756 (1971).

[12] I. Martin, C. Lavin, C. Barrientos, Can. J. Phys. 69, 1273 (1991).

[13] C. Lavin, C. Barrientos, I. Martin, J. Quant. Spectrosc. Radiat. Transfer 47, 411 (1992).

[14] A. Kramida, Yu. Ralchenko, J. Reader, and NIST ASD Team, 2014, NIST Atomic Spectra Database, ver. 5.2, National Institute of Standards and Technology, Gaithersburg (MD) 2015.

[15] G. Çelik, H.Ş. Kılıc, E. Akın, Turk. J. Phys. 30, 165 (2006).

[16] G. Çelik, E. Akın, H.Ş. Kılıc, Eur. Phys. J. D 40 , 325 (2006).

[17] G. Çelik, E. Akın, H.Ş. Kılıc, Int. J. Quant. Chem. 107, 495 (2007).

[18] Ş. Ateş, G. Çelik, Acta Phys. Pol A 116, 169 (2009).

[19] M. Yıldız, G. Çelik, H.Ş. Kılıc, Acta Phys. Pol A 115, 641 (2009).

[20] G. Çelik, Ş. Ateş, G. Tekeli, Can. J. Phys. 94, 23 (2016).

[21] U.I. Safronova, M.S. Safronova, Phys. Rev. A 87, 032502 (2013).

[22] C. Froese Fischer, M. Saparov, G. Gaigalas, M. Godefroid, At. Data Nucl. Data Tables 70, 119 (1998).

[23] C.E. Theodosiou, Phys. Scr. 45, 450 (1992).

[24] I.L. Glukhov, E.A. Nikitina, V.D. Ovsiannikov, Phys. Scr. T157, 014014 (2013).

[25] I.L. Glukhov, E.A. Nikitina, V.D. Ovsiannikov, Opt. Spectrosc. 115, 1 (2013).

[26] Information system Electronic structure of atoms, Novosibirsk State University, Institute of Automation and Electrometry.

[27] A. Lindgard, S.E. Nielsen, At. Data Nucl. Data Tables 19, 533 (1977).

[28] J. Bromander, Phys. Scr. 4, 61 (1971).

[29] S. Hontzeas, I. Martinson, P. Erman, R. Buchta, Phys. Scr. 6, 55 (1972).

[30] T. Andersen, K.A. Jessen, G. Sorensen, Phys. Rev. 188, 76 (1969).

[31] R. Kurucz, http://kurucz.harvard.edu/atoms/ 0401/1ife0401. dat. 